\title{
Introduction : Le Nord-Est français, un espace viticole paradoxal et dynamique
}

Introduction: French north-eastern, a paradoxical and dynamic vineyard area Einleitung: Der Nordssten Frankreichs, ein paradoxes Weinbaugebiet und dynamisches

\section{Gabriel Wackermann}

\section{(2) OpenEdition}

\section{Journals}

Édition électronique

URL : http://journals.openedition.org/rge/894

DOI : $10.4000 /$ rge.894

ISSN : 2108-6478

Éditeur

Association des géographes de l'Est

Édition imprimée

Date de publication : 1 janvier 2004

Pagination : 5-7

ISSN : 0035-3213

\section{Référence électronique}

Gabriel Wackermann, «Introduction : Le Nord-Est français, un espace viticole paradoxal et

dynamique », Revue Géographique de l'Est [En ligne], vol. 44 / 1-2 | 2004, mis en ligne le 28 mai 2009, consulté le 08 septembre 2020. URL : http://journals.openedition.org/rge/894; DOI : https://doi.org/ $10.4000 /$ rge.894

Ce document a été généré automatiquement le 8 septembre 2020.

Tous droits réservés 


\section{Introduction : Le Nord-Est français, un espace viticole paradoxal et dynamique}

Introduction: French north-eastern, a paradoxical and dynamic vineyard area

Einleitung: Der Nordssten Frankreichs, ein paradoxes Weinbaugebiet und dynamisches

\section{Gabriel Wackermann}

1 Le Nord-est français est soumis, en ce qui concerne la viticulture, aux contraintes qui frappent son territoire : la vigne prospère réellement entre les $28^{\circ}$ et $50^{\circ}$ degrés de latitude, mais les variantes climatiques locales, le goût changeant des consommateurs au fil des siècles ont contribué tant aux avancées vers le nord qu'aux tribulations d'une vigne qui n'est pas réellement à son aise au-delà de l'aire "naturelle ». Si d'une part les pays froids sont souvent caractérisés par des vins acides et aigrelets, nous observons d'autre part que des vignobles de qualité se sont développés et affirmés mondialement au-delà des limites de culture considérées comme "normales»: Champagne, Bourgogne Franche-Comté, et Alsace notamment apparaissent comme des témoins éloquents de la victoire de l'homme et des sociétés viticoles concernées sur les aléas hivernaux, les gels printaniers, la pluviosité exceptionnelle, le ravinement des coteaux, l'ensoleillement insuffisant, les menaces automnales pesant sur les vendanges, les défauts initiaux de la qualité des vins, ...Les énergies déployées pour conjurer le mauvais sort, le professionnalisme, l'effort permanent en matière d'affinement et d'adaptation à un marché à la fois qualitatif et compétitif ont permis de réussir une performance extraordinaire: une grande finesse des vins et une large gamme de variétés subtiles donnant dans la nuance.

2 Dans sa contribution, Jean-Pierre Chabin parle du "paradoxe retourné», évoquant comme explication tout particulièrement "le génie humain", selon Roger Dion (Le paysage et la vigne..., Paris, Payot, réédition de 1990), ainsi que « la synthèse entre des atouts naturels qui existent et qui contrebalancent les risques et une remarquable 
adaptation des hommes aux contraintes naturelles» (Rolande Gadille (Le vignoble de la Côte bourguignonne..., Paris, Les Belles Lettres, 1967). Il prend résolument position pour cette dernière approche, ajoutant toutefois « que la part respective de l'élément naturel et de l'élément humain est... impossible à fixer ». Il affine davantage en rappelant le vieil adage du vigneron bourguignon, selon lequel la vigne doit souffrir « pour produire l'excellence. Ceci revient à instituer la limite comme un élément constitutif de la qualité, dans la mesure où l'Homme en domine les contraintes et en assume les risques ». Cette action volontariste et imaginative de la société viticole, cette « culture à la limite (qui) a suscité l'innovation", est valable pour bien d'autres vignobles septentrionaux, « la plus célèbre par la valeur ajoutée exceptionnelle qu'elle confère au produit étant celle de la méthode de champagnisation », selon Chabin.

3 Ce sont tout particulièrement les terroirs viticoles champenois qui conduisent Alain Marre a insister lui aussi sur le fait que la localisation du vignoble s'explique tant par des conditions naturelles que par des caractéristiques historiques. Tradition et terroir sont étroitement liés, ce dernier n'étant pas que le fruit de la nature, mais aussi et surtout celui du travail des hommes, qui créent des traditions et n'oublient pas de s'adapter aux évolutions. La Champagne offre l'exemple d'un vignoble «froid» septentrional (Marre), conquis sur la nature par des pratiques culturales appropriées, et façonné en fonction des potentialités naturelles de base. Le champagne produit ainsi depuis le XVI ${ }^{e}$ siècle sur un terroir aux multiples " unités homogènes de terroirs ", a donné lieu à des emboîtements d'échelles qui favorisent la manière française de cultiver la vigne et les zones d'appellation contrôlée, contrairement aux vins de Californie ou de l'hémisphère austral, qui relèvent avant tout du cépage. En France même, la main de l'homme a diversifié jusqu'à l'image des lieux de production, symbolisée par la bouteille: tandis qu'en Champagne ce sont les Maisons de Champagne qui font référence, le Bordelais se singularise par ses Châteaux...

4 Partant des fondements physiques de la viticulture mâconnaise, Gérard Mottet relève l'originalité du relief très fracturé par la tectonique qui compose le vignoble de ce pays, ayant donné lieu à 43 appellations communales AOC, ce qui a rendu indispensable des propositions de regroupements dans les appellations. Les multiples petites unités physiques, l'analyse des usages, structures et AOC du Mâconnais conduisent l'auteur à dire «combien il est important que la géographie soit présente pour rappeler l'impérative rigueur des milieux physiques dans ses rapports avec les "produits de terroirs" ». Si l'auteur met en outre l'accent sur le fait qu'« un travail d'esprit réellement géographique s'avérait nécessaire » pour «définir ce qu'est réellement le Mâconnais viticole..., puis à caractériser, en fonction de la complexité des milieux, les petites unités physiques permettant ensuite de présenter des regroupements d'appellations communales ", il n'omet pas ce " génie humain » qui, dès la préhistoire, a conféré à la Bourgogne "ce rôle essentiel de liant, d'“axe de civilisation" ", auquel fut intégré dès l'Antiquité romaine le commerce du vin.

5 Braver le destin, la nature en somme, s'y accommoder et la surpasser par une ténacité sans limites, fut payante. Ce comportement a donné naissance à une diversité extraordinaire de productions : « ...si la Bourgogne, avec sa pléiade de climats, morcelés en restrictifs "grands" et "premiers crus", ressemble à un émail champlevé aux cloisons innombrables... l'Alsace quant à elle n'exprime bien son particularisme qu'à travers ses centaines de microclimats, encore aggravés dans la multiplicité de leurs productions par la diversité des cépages. A telle enseigne qu'il fallut attendre 1970, soit plus de 
trente années après la grande loi sur les appellations d'origine pour que le Législateur parvînt à proposer une logique organique dans cette tapisserie au petit point, apparemment complexe mais qui n'a rien d'abstrait » (Bernard Ginestet, préface à Alsace clos et grands crus, Paris, J. Legrand, 1989).

L'ampleur même des résultats obtenus a été fonction d'un long cheminement, caractérisé par un labeur harassant, aux tribulations nombreuses, quasiment deux fois millénaires. L'entreprise romaine d'extension pionnière et fondamentale du vignoble méditerranéen, d'essence principalement culturelle, fut suivie de celle, liturgique, de l'Église. La christianisation et le vignoble allaient de pair jusqu'aux limites septentrionales extrêmes des potentialités de production du vin: vin de messe et vin festif accompagnaient « les heurs et les jours » de sociétés à la fois orientées vers le ciel et fortement ancrées dans les libations terrestres.

Des traditions tenaces ont contribué à sauvegarder la vigne en-dehors de ses limites climatiques «normales». Ainsi la loi salique des Francs a interdit son arrachage. Charlemagne a encouragé les plantations et créé de nouveaux vignobles.

Lorsque l'invasion musulmane, au VIII ${ }^{\mathrm{e}}$ siècle, a fait de la Méditerranée une mer arabe, elle a mis fin aux grands trafics maritimes et aux échanges commerciaux. Dans les "pays du nord", de gros efforts furent alors entrepris par les puissants - ceux du pouvoir politique et ecclésiastique, les détenteurs de capitaux- en vue de l'acquisition de vignobles. Les institutions monastiques firent en outre appel à la piété et/ou au sens diplomatique des souverains pour se faire octroyer de prometteuses terres à vigne. Des legs non moins pieux vinrent compléter le patrimoine viticole déjà si riche de l'Eglise.

En dépit du fait que dès le $\mathrm{X}^{\mathrm{e}}$ siècle les transports de vins reprirent de plus belle, la pression croissante des pays du nord sur la demande contribua à la progression de la vigne en direction des coteaux septentrionaux, même sur des territoires où celle-ci avait disparu. Les vins verts et acides n'étaient pas redoutés autant que de nos jours. Ainsi, à la fin du Moyen-Âge - Roger Dion l'a précisé - , la limite septentrionale de la vigne dépassait largement celle à laquelle nous sommes habitués de nos jours : les vins des vallées du Rhin, de la Moselle et de la Meuse, des pays rhénans en somme, furent acheminés par la voie fluviale vers la Hollande, voire l'Angleterre. Cologne fut un centre majeur du commerce international du vin. La Hanse servit d'intermédiaire jusque sur les rives de la Baltique.

10 Si la notion de "vin de Champagne " n'était pas connue à cette époque-là, cette province comportait toutefois déjà de nombreux vignobles: Reims, Epernay, Vertus, Châlons p. ex. furent des centre viticoles connus et importants. Lors du déroulement des foires de Champagne, les draps flamands et ceux du Hainaut ont été troqués contre les vins de cette province.

11 La Bourgogne, encore relativement bien placée dans la zone d'extension de la vigne vers le nord, parvint à surmonter les difficultés de transport vers les aires septentrionales dès le XIII ${ }^{e}$ siècle, à la faveur de l'ascension des ducs de Bourgogne et à la qualité des grands crus de la Côte d'Or. Au XIII ${ }^{e}$ siècle aussi, la Franche-Comté, par Arbois et Poligny interposées, s'est insérée dans la compétition en direction du nord. Le « Registrium Ville Bellifortis » atteste la présence de la vigne par un document daté du 8 avril 1432, qui signale que trois bourgeois belfortins ont obtenu l'autorisation de mettre une pièce de terre en vigne. 
12 Roland Nistri et Claude Prêcheur nous signalent (La région du nord et du nord-est, Paris, PUF, 1959) qu'après la Seconde guerre mondiale, époque à laquelle l'agriculture lorraine était toujours essentiellement vivrière, les plus fortes densités rurales se manifestaient dans les villages viticoles. Ils constatent cependant qu' « à partir de la fin $\mathrm{du}\left(\mathrm{XIX}^{\mathrm{e}}\right)$ siècle, le vignoble était sérieusement menacé par la concurrence des vins du Midi » et que, dans de nombreux cas, il ne devait pas survivre à la Grande guerre.

Le grand problème demeure, lié à la survie viticole du «nord»: la qualité. En Alsace tout particulièrement, la Révolution a favorisé la multiplication des petits propriétaires. Il fallut attendre la période de l'Entre-Deux-Guerres pour y rattraper un siècle de retard sur les autres régions françaises. Aussi, sous l'impulsion de quelques viticulteurs éclairés, le mouvement de requalification prit-il une allure accélérée dans le cadre d'une politique générale de développement et d'aménagement du territoire.

14 Ainsi, dans une région aussi sollicitée que l'Alsace viticole par l'urbanisation, depuis l'après-Seconde guerre mondiale, le vignoble en voie de qualification et de reconnaissance internationale, a servi de front «non aedificandi», d'arrêt à la spéculation immobilière tendant à ravager les paysages parmi les plus typiques de cette région (Gabriel Wackermann: "Les terres à vigne alsaciennes suscitent-elles des situations conflictuelles?», La protection des terroirs agricoles, CERVIN, Université de Bordeaux III, 1993, pp. 91-102).

15 Dans cette optique, de multiples efforts ont été et continuent à être accomplis. Caroline Grégoire et Claire Litaudon-Jouve nous expliquent, à l'exemple de trois communes basrhinoises - Scherwiller, Rosenwiller et Rosheim -, la dynamique de l'occupation des sols en zone viticole. Elles constatent comment les effets conjoints de l'augmentation des surfaces en vigne associée à la diminution du nombre d'exploitations induit une modification de l'occupation du sol, une augmentation du bâti, essentiellement au détriment des surfaces en prairies et cultures, de même que des surfaces en vergers. Pour connaître à fond le fonctionnement de ces mécanismes à l'échelle communale, elles ont eu recours à l'analyse de photographies aériennes et à des statistiques couvrant la période allant de 1976 à 1998. L'étude confirme que le vignoble du piémont vosgien «est un milieu particulier caractérisé par de très forts enjeux, aussi bien économiques, sociaux qu'environnementaux qui se sont exprimés de diverses façons tout au long de son histoire ". Elle révèle aussi que l'observation suivie à l'échelle communale, surtout en ce qui concerne l'évolution du vignoble, est indispensable. Malheureusement, " aucun outil testé ne s'est révélé le plus pertinent ». La solution ne pourra être trouvée qu'« avec l'évolution de la technologie et la disponibilité d'images à très haute résolution ».

16 Caroline Grégoire et Julien Tournebize démontrent que l'enherbement du vignoble alsacien accuse un bilan positif en matière de transfert de nitrates, la présence d'herbe étant source de fertilisation et de mobilisation de l'azote sous forme organique, suscitant une réduction des flux d'azote jusqu'à un facteur 7. L'enherbement est devenu un véritable enjeu régional, sous réserve d'une régulation en fonction de la quantité d'eau disponible, contrôlée par la pluviométrie. La télédétection a révélé qu'en 1999 plus des trois quarts du territoire viticole était enherbé. En 2001/2002, des chercheurs ont pu constater que 13 autres pourcents étaient encore potentiellement enherbables.

17 Sylvaine Boulanger-Fassier aborde, de son côté, la question de cette multitude de petits et très petits vignerons alsaciens et jurassiens qui, en limitant les rendements et en cultivant des cépages nobles, tout en organisant systématiquement l'écoulement de 
leur production par le truchement de coopératives, sont parvenus à faire face à une demande fluctuante et à une âpre concurrence nationale et internationale. Dans le cadre d'une concentration générale favorisant l'extension des superficies viticoles, les deux régions s'appuient, l'alsacienne sur la partie centrale de son espace viticole, la franc-comtoise sur le pôle arboisien. Les deux personnalités divergent néanmoins à maint égard : «En Alsace, le lien entre le produit et le paysage est fort, tandis qu'il est souvent inexistant dans le Jura, ... ». Les notions d'accueil et de séjour, de route des vins, de proximité internationale répondent à des paramètres différents. Le vignoble alsacien est un pôle d'attraction, "contrairement à son homologue jurassien ", écrit l'auteur. Mais le marché mondial demeure plus menaçant que jamais.

Si la poussée de l'urbanisation n'a pas empêché l'essor viticole de multiples secteurs ruraux, elle a néanmoins été préjudiciable à la vigne dans les aires métropolitaines densément occupées par les hommes et leurs activités non agricoles. Jean-Pierre Husson illustre cette évolution en s'appuyant sur l'exemple de l'agglomération nancéienne, dans laquelle la première couronne urbaine comporte les anciens coteaux viticoles. Dans une démarche très expressive, il nous conduit « de l'objet relique au projet de renaturation ». Il demeure très positif dans l'appréciation des "héritages fossilisés, longtemps enfrichés» qui "offrent d'intéressantes possibilités pour oser articuler des formes assez variées de cohabitations de la nature et de la ville». Du cas métropolitain lorrain à celui, évoqué ci-dessus, de l'Alsace des petites et moyennes villes viticoles, plus ou moins garantes de la préservation paysagère, nous constatons que la vigne demeure inscrite d'une façon ou d'une autre dans l'urbanisme contemporain. Les riches passé et présent viticoles demeurent partie intégrante des préoccupations en matière d'aménagement.

\section{AUTEUR}

\section{GABRIEL WACKERMANN}

Professeur émérite des Universités, Président de l'Association de Géographes de l'Est, 180 route de Oberhausbergen, 67000 Strasbourg 